\title{
A UNIFORM ALGEBRA OF ANALYTIC FUNCTIONS ON A BANACH SPACE
}

\author{
T. K. CARNE, B. COLE AND T. W. GAMELIN
}

\begin{abstract}
Let $A(B)$ be the uniform algebra on the unit ball of a dual Banach space $\mathscr{Z}=\mathscr{Y}^{*}$ generated by the weak-star continuous linear functionals. We focus on three related problems: (i) to determine when $A(B)$ is a tight uniform algebra; (ii) to describe which functions in $H^{\infty}(B)$ are approximable pointwise on $B$ by bounded nets in $A(B)$; and (iii) to describe the weak topology of $B$ regarded as a subset of the dual of $A(B)$. With respect to the second problem, we show that any polynomial in elements of $\mathscr{Y}^{* *}$ can be approximated pointwise on $B$ by functions in $A(B)$ of the same norm. This can be viewed as a generalization of Goldstine's theorem. In connection with the third problem, we introduce a class of Banach spaces, called $\Lambda$-spaces, with the property that if $\left\{x_{j}\right\}$ is a bounded sequence in $\mathscr{X}$ such that $P\left(x_{j}\right) \rightarrow 0$ for any $m$ homogeneous analytic function $P$ on $\mathscr{X}, m \geq 1$, then $x_{j} \rightarrow 0$ in norm. We show for instance that a Banach space has the Schur property if and only if it is a $\Lambda$-space with the Dunford-Pettis property.
\end{abstract}

\section{INTRODUCTION}

We are interested in studying certain algebras of analytic functions on a Banach space. Our motivating problem is to determine when these algebras are tight. We obtain some partial results, and this leads along the way to a number of other problems involving Banach spaces and the analytic functions thereon.

We fix a (complex) Banach space $\mathscr{Y}$, with dual space $\mathscr{Z}=\mathscr{Y}^{*}$. We will focus on one specific uniform algebra $A(B)$ associated with $\mathscr{Y}$, defined to be the algebra generated by the weak-star continuous linear functionals on the closed unit ball $\bar{B}$ of $\mathscr{Z}$. In other words, $A(B)$ is the uniform closure on $\bar{B}$ of polynomials in the elements of $\mathscr{Y}$, regarded as functions on the weak-star compact set $\bar{B}$.

The functions in $A(B)$ are clearly analytic on $B$. It is easy to see that the spectrum of $A(B)$ coincides with $\bar{B}$. Arenson [1] has shown that the strong boundary points of $A(B)$ are precisely the complex extreme points of $\bar{B}$. Arenson also described the Gleason parts of $A(B)$, in a more general setting.

There are three types of questions which we shall focus on, and which are related. The first problem is to determine when $A(B)$ is a tight uniform algebra

Received by the editors December 5, 1988.

1980 Mathematics Subject Classification (1985 Revision). Primary 46J15; 46B10.

The second author was supported by NSF grant \#DMS86-12012.

The third author was supported by NSF grant \#DMS85-03780. 
on $\bar{B}$. The notion of tightness was introduced in [2]. It corresponds to the solvability of a certain $\bar{\partial}$-problem with sup-norm estimates and a mild gain in smoothness. We conjecture that if $A(B)$ is tight, then $\mathscr{Z}$ is finite dimensional. We are able to show that $A(B)$ is not tight for many Banach spaces, such as the nonreflexive Banach spaces, and the $L^{p}$-spaces.

In developing criteria for tightness we are led to study the weak topology of $B$ as a subset of the dual of $A(B)$, that is, the $A(B)^{* *}$-topology of $B$. One can describe the $A(B)^{* *}$-topology on $B$ as the weak topology with respect to a certain algebra of bounded analytic functions on $B$, namely, the algebra $A_{p b}(B)$ of functions in $H^{\infty}(B)$ which can be approximated pointwise on $B$ by bounded nets in $A(B)$. This leads to our second problem, to determine when $A_{p b}(B)$ coincides with $H^{\infty}(B)$. We will show that if $\mathscr{Z}$ has the metric approximation property, then every $f \in H^{\infty}(B)$ is the pointwise limit of a net of functions in $A(B)$ with the same norm as $f$. Along the way we extend Goldstine's theorem on the approximation of linear functionals on the unit ball of a dual Banach space by weak-star continuous functionals, obtaining the analogous approximation result for polynomials in linear functionals by polynomials in weak-star continuous linear functionals.

Our third problem is to understand the $A(B)^{* *}$-topology of $B$. We show that the $A(B)^{* *}$-topology of $B$ does not coincide with the norm topology unless $\mathscr{Z}$ is finite dimensional. On the other hand, for certain Banach spaces $\mathscr{Z}$ we show that the $A(B)^{* *}$-convergent sequences in $B$ are norm convergent. This type of result can be regarded as a variant of Schur's theorem, that the weakly convergent sequences in $l^{1}$ are norm convergent.

The ideas related to the Schur property are treated in the context of an arbitrary Banach space. We define a Banach space $\mathscr{Z}$ to be a $\Lambda$-space if whenever $\left\{x_{j}\right\}$ is a sequence in $\mathscr{X}$ such that $f\left(x_{j}\right) \rightarrow 0$ for every $m$-homogeneous analytic function $f$ on $\mathscr{X}$, for any $m \geq 1$, then $\left\|x_{j}\right\| \rightarrow 0$. The spaces $l^{p}, 1 \leq p<\infty$, are $\Lambda$-spaces, as are the spaces $L^{p}[0,1]$ for $2 \leq p<\infty$. The spaces $C(X)$ and $L^{1}[0,1]$ are not $\Lambda$-spaces. We show that if $\mathscr{X}$ has the Dunford-Pettis property, then the weak topology of $\mathscr{X}$ coincides on any weakly compact set with the "weak polynomial topology", the topology of pointwise convergence for all $m$-homogeneous analytic functions, $n \geq 1$. It follows that $\mathscr{X}$ has the Schur property if and only if $\mathscr{X}$ is a $\Lambda$-space with the DunfordPettis property.

The paper is organized as follows. $\S \S 2$ and 3 contain some preliminary observations and $\S 4$ is devoted to pointwise bounded approximation. The $A(B)^{* *}$ topology is discussed in $\S 5$. $\S \S 6$ and 7 are devoted to $\Lambda$-spaces. In $\S 8$ we sharpen some results from [2] on tight algebras, and in $\S 9$ we consider the problem of when $A(B)$ is tight.

For background information on uniform algebras see [7], and for the bidual of a uniform algebra see [3] or [8]. Our reference for analytic functions on a Banach space is [11]. 
We will denote by $\mathscr{X}$ an arbitrary Banach space. The open unit ball of $\mathscr{X}$ will be denoted by ball $\mathscr{X}$, or by $B$ when no confusion can arise. The closed unit ball is denoted by $\bar{B}$. The algebra of bounded analytic functions on $B$ is denoted by $H^{\infty}(B)$. For $m \geq 1, \mathscr{P}_{m}(\mathscr{X})$ will denote the $m$-homogeneous analytic functions on $\mathscr{X}$. Thus $\mathscr{P}_{1}(\mathscr{X})$ is the dual $\mathscr{X}^{*}$ of $\mathscr{X}$. We denote by $\mathscr{P}(\mathscr{X})$ the algebra of analytic polynomials, that is, finite linear combinations of elements of the $\mathscr{P}_{m}$ 's. Finally, we will use upon occasion the pointed bracket $\langle\cdot, \cdot\rangle$ for the pairing between a Banach space and its dual, in no particular order.

\section{Preliminaries on $H^{\infty}(B)$}

In this section we note for future reference certain facts about the algebra $H^{\infty}(B)$ of bounded analytic functions on the unit ball $B$ of an arbitrary Banach space $\mathscr{X}$.

The functions in $H^{\infty}(B)$ satisfy a Schwarz-type estimate of the form

$$
|f(x)-f(y)| \leq 2\|f\|_{B}\|x-y\| /(1-\|x\|), \quad x, y \in B .
$$

Indeed, if $g$ is analytic on a ball in $\mathscr{X}$ with center $x$ and radius $\delta>0$, and $g(x)=0$, then the Schwarz inequality yields

$$
|g(y)| \leq\|g\|\|y-x\| / \delta, \quad\|y-x\|<\delta .
$$

Applying this estimate to $f-f(x)$, with $\delta=1-\|x\|$, we obtain (2.1) above.

The estimate (2.1) shows in particular that the functions in $H^{\infty}(B)$ are uniformly continuous on any fixed subball $r B, 0<r<1$, of $B$.

Let $f \in H^{\infty}(B)$. For each $x \in \mathscr{X}$, the function $f(\lambda x)$ has a power series expansion

$$
f(\lambda x)=\sum_{m=0}^{\infty} f_{m}(x) \lambda^{m},
$$

converging normally for $|\lambda|<1 /\|x\|$. The coefficient functions $f_{m}$ belong to $\mathscr{P}_{m}(\mathscr{X})$ and satisfy $\left\|f_{m}\right\|_{B} \leq\|f\|_{B}$. We refer to $f \sim \sum f_{m}$ as the Taylor series expansion of $f$. In view of the uniform continuity of $f$ on each ball $r B, 0<r<1$, we obtain easily the following lemma as a consequence of the corresponding results for analytic disks.

2.1. Lemma. Let $f \in H^{\infty}(B)$ have power series expansion $f \sim \sum f_{m}$. Then the series $\sum f_{m}$ converges uniformly to $f$ on each fixed ball $r B, 0<r<1$. Furthermore, if $\left\{g_{m}\right\}$ is the sequence of Cesàro means of the partial sums of the series for $f$, then

$$
\left\|g_{m}\right\|_{B} \leq\|f\|_{B},
$$

and $g_{m}$ converges uniformly to $f$ on each ball $r B, 0<r<1$.

\section{The bidual of $A(B)$}

Now we turn to the fixed Banach space $\mathscr{Y}$ and its dual $\mathscr{Z}$. The open unit ball of $\mathscr{Z}$ will be denoted here by $B$. Recall that $A(B)$ is the uniform algebra 
on $\bar{B}$ generated by the weak-star continuous linear functionals, while $A_{p b}(B)$ is the algebra of functions on $B$ which are approximable pointwise by bounded nets in $A(B)$. For $f \in A_{p b}(B)$ we define the triple-norm $\|f\| \|$ to be the infimum of constants $c>0$ such that there is a net is in cball $A(B)$ converging pointwise to $f$ on $B$. Thus for each $f \in A_{p b}(B)$ there is a net $\left\{f_{\alpha}\right\}$ in $A(B)$ satisfying

$$
\left\|f_{\alpha}\right\|_{B} \leq\|\| f\|\|, \quad f_{\alpha}(z) \rightarrow f(z), \quad z \in B .
$$

On account of the uniform boundedness of the $f_{\alpha}$ 's, they converge normally on every analytic disk in $B$, so that $f$ is analytic on any analytic disk in $B$. Since $f$ is bounded, it is analytic on $B$, and

$$
A_{p b}(B) \subseteq H^{\infty}(B) \text {. }
$$

The algebra $A_{p b}(B)$ is closely related to the bidual $A(B)^{* *}$ of $A(B)$. Indeed, the point evaluations at points of $B^{\prime}$ extend weak-star continuously to $A(B)^{* *}$, so that the functions $F \in A(B)^{* *}$ determine point functions $\left.F\right|_{B}$ on $B$. We claim that

$$
\left.A(B)^{* *}\right|_{B}=A_{p b}(B),
$$

and that

$$
\||f|\|=\inf \left\{\|F\|: F \in A(B)^{* *},\left.F\right|_{B}=f\right\}, \quad f \in A_{p b}(B) .
$$

Indeed by Goldstine's theorem any $F \in A(B)^{* *}$ is weak-star approximable by a net $\left\{f_{\alpha}\right\}$ in $A(B)$ satisfying $\left\|f_{\alpha}\right\|_{B} \leq\|F\|_{A(B)^{* *}}$. The net $\left\{f_{\alpha}\right\}$ converges pointwise to $\left.F\right|_{B}$ on $B$, so that $\left.F\right|_{B} \in A_{p b}(B)$ and $\left\||F|_{B}\right\| \mid \leq\|F\|_{A(B)^{* *}}$. On the other hand, if $f \in H^{\infty}(B)$ is a pointwise limit of a net in $A(B)$ bounded by $\||f|\|$, and if $F$ is a weak-star adherent point of the net in $A(B)^{* *}$, then $\left.F\right|_{B}=f$, and $\|F\|_{A(B) *} \leq\|\| f\|\|$.

Our main objective in this section is to record the following observation concerning the power series expansion of functions in $A_{p b}(B)$.

3.1. Theorem. Suppose $f \in H^{\infty}(B)$ has Taylor series expansion $f \sim \sum f_{m}$. If $f$ belongs to $A_{p b}(B)$, then so does $f_{m}$ for all $m \geq 0$, and

$$
\left\|f_{m}\right\| \leq\|\| f\|\|, \quad m \geq 0 .
$$

Conversely, if each $f_{m}$ belongs to $A_{p b}(B)$, and if the sequence $\left\{g_{m}\right\}$ of Cesàro means of the partial sums of $\sum f_{m}$ satisfies

$$
\sup _{m}\left\|\left|g_{m}\right|\right\|<\infty
$$

then $f \in A_{p b}(B)$.

Proof. Let $f \in A_{p b}(B)$. Let $\left\{f_{\alpha}\right\}$ be a net in $A(B)$ converging pointwise to $f$ on $B$, such that $\left\|f_{\alpha}\right\|_{B} \leq\|\| f\|\|$. Let $f_{\alpha}$ have Taylor expansion $\sum f_{\alpha m}$. Fix $x \in$ $\mathscr{X}$ with $\|x\| \leq 1$. From the expansion (2.2) for $f(\lambda x)$, and the corresponding 
expansion for $f_{\alpha}(\lambda x)$, we see that the coefficients $f_{\alpha m}(x)$ converge to $f_{m}(x)$ for each fixed $m \geq 0$. Furthermore,

$$
\left|f_{\alpha m}(x)\right| \leq\left\|f_{\alpha}\right\|_{B} \leq\||| f \mid\|,
$$

so that

$$
\left\|f_{\alpha m}\right\|_{B} \leq\||| f \mid\|,
$$

and

$$
\left\|\left|f_{m}\right|\right\| \leq|\||f|\| .
$$

This proves the first statement.

For the converse, suppose the Cesàro means $g_{m}$ belong to $A_{p b}(B)$ and satisfy $\left\|\left|g_{m}\right|\right\| \leq c, m \geq 1$. Let $\varepsilon>0$ and let $S$ be a finite subset of $B$. By choosing $m$ so large that $\left|g_{m}-f\right|<\varepsilon$ on $S$, and then finding $h_{S, \varepsilon} \in A$ such that $\left\|h_{S, \varepsilon}\right\| \leq c$ and $\left|h_{S, \varepsilon}-g_{m}\right|<\varepsilon$ on $S$, we obtain a net $\left\{h_{S, \varepsilon}\right\}$ which converges pointwise boundedly to $f$ on $B$.

There are two natural questions which arise. The first asks for which $\mathscr{Z}$ is $A_{p b}(B)=H^{\infty}(B)$ ? The second is more technical, and asks when $H^{\infty}(B)$ is a direct summand of $A(B)^{* *}$. In terms of the minimal idempotent $P_{B}$ associated with the Gleason part $B$ (cf. [8]), this problem boils down to determining when

$$
P_{B} A(B)^{* *} \cong H^{\infty}(B) \text {. }
$$

We address the first of these questions in the next section.

\section{A generalization of Goldstine's theOREM}

In this section we aim to give a criterion under which $A_{p b}(B)=H^{\infty}(B)$. First we wish to establish the following approximation theorem, which can be viewed as an extension of Goldstine's theorem that ball $\mathscr{Y}$ is weak-star dense in ball $\mathscr{Y}^{* *}$.

4.1. Theorem. Let $B$ be the open unit ball of the dual Banach space $\mathscr{Z}=\mathscr{Y}^{*}$. Let $f$ be a (finite) polynomial in elements of $\mathscr{Y}^{* *}$. Then there is a net $\left\{f_{\alpha}\right\}$ of polynomials in elements of $\mathscr{Y}$ such that

$$
\left\|f_{\alpha}\right\|_{B} \leq\|f\|_{B}, \quad \text { all } \alpha, \quad \lim f_{\alpha}(z)=f(z), \quad \text { all } z \in B .
$$

The proof will be momentarily postponed. First we make some remarks.

Let $A_{*}(B)$ denote the algebra of uniform limits on $B$ of polynomials in elements of $\mathscr{Y}^{* *}$, i.e., $A_{*}(B)$ is the closed subalgebra of $H^{\infty}(B)$ generated by $\mathscr{Y}^{* *}$. Because of the sharp estimate for the norms of the $f_{\alpha}$ 's, one can also approximate pointwise on $B$ any function in $A_{*}(B)$. Thus Theorem 4.1 can be restated as asserting that ball $A(B)$ is dense in ball $A_{*}(B)$, in the topology of pointwise convergence on $B$. In particular, $A_{*}(B) \subseteq A_{p b}(B)$.

From the proof of Theorem 4.1 it will be seen that the $f_{\alpha}$ 's can be chosen to have the same degree as $f$. This can also be deduced directly from the statement of Theorem 4.1, by passing to the Cesàro means of partial sums associated with an arbitrary approximating net. 
If $f$ is homogeneous, then the $f_{\alpha}$ 's can be chosen to be homogeneous of the same degree as $f$. Again this can be seen from the proof, or it can be deduced directly from Theorem 4.1 by taking the appropriate term in the Taylor series of an arbitrary approximating net. Thus for instance if $f$ is linear, then the $f_{\alpha}$ 's can be chosen to be linear, and the conclusion of Theorem 4.1 reduces to be Goldstine's theorem.

The proof of Theorem 4.1 will depend on the weak-star density of the unit ball of $\mathscr{B}(E, \mathscr{X})$ in that of $\mathscr{B}(E, \mathscr{X})^{* *}$, whenever $E$ is finite dimensional. This is Goldstine's theorem, applied to $\mathscr{B}(E, \mathscr{X})$, together with the equation

$$
\mathscr{B}(E, \mathscr{X})^{* *} \cong \mathscr{B}\left(E, \mathscr{X}^{* *}\right) \text {. }
$$

For a proof of this equation, see the paper of Dean [4], where a short proof of the related "principle of local reflexivity" is given based on the equation. (See also [9, 13].) A dual version of the density statement is as follows.

4.2. Lemma. Let $\mathscr{X}$ be a Banach space, and let $E$ be a finite dimensional Banach space. Let $T$ be a continuous linear operator from $\mathscr{X}^{*}$ to $E$. Then there is a net $\left\{T_{\alpha}\right\}$ of continuous linear operators from $\mathscr{X}^{*}$ to $E$ such that

(i) $T_{\alpha}(z) \rightarrow T(z)$ for all $z \in \mathscr{X}^{*}$,

(ii) $T_{\alpha}$ (ball $\left.\mathscr{X}^{*}\right) \subseteq T$ (ball $\mathscr{X}^{*}$ ) for all $\alpha$,

(iii) each $T_{\alpha}$ is continuous with respect to the weak-star topology of $\mathscr{X}^{*}$.

Proof. Replacing $E$ by the range of $T$, we can assume that $T\left(\mathscr{X}^{*}\right)=E$. Replacing the norm on $E$ by an equivalent norm, we can assume that $T$ (ball $\mathscr{X}^{*}$ ) coincides with ball $E$, so that in particular $\|T\|=1$. The statement (ii) then becomes

(ii) $^{\prime}\left\|T_{\alpha}\right\| \leq 1$, all $\alpha$.

Now we apply Goldstine's theorem, to approximate $T^{*} \in \mathscr{B}\left(E^{*}, \mathscr{X}^{* *}\right) \cong$ $\mathscr{B}\left(E^{*}, \mathscr{X}\right)^{* *}$ by a net $\left\{S_{\alpha}\right\}$ in $\mathscr{B}\left(E^{*}, \mathscr{X}\right)$, so that $\left\|S_{\alpha}\right\| \leq 1$ and $\left\langle S_{\alpha} \varphi, z\right\rangle$ tends to $\left\langle T^{*} \varphi, z\right\rangle$ for all $\varphi \in E^{*}$ and $z \in \mathscr{X}^{*}$. Set $T_{\alpha}=S_{\alpha}^{*}$, regarded as an operator from $\mathscr{X}^{*}$ to $E$. Then (ii) ${ }^{\prime}$ and (iii) are valid, and furthermore

$$
\lim \left\langle\varphi, T_{\alpha} z\right\rangle=\langle\varphi, T z\rangle, \quad \varphi \in E^{*}, z \in \mathscr{X}^{*},
$$

from which (i) follows.

Proof of Theorem 4.1. By hypothesis, $f$ is a finite linear combination of monomials of the form $\varphi_{1}(z) \ldots \varphi_{k}(z)$, where $\varphi_{1}, \ldots, \varphi_{k} \in \mathscr{X}^{* *}$. Let $\varphi_{1}, \ldots, \varphi_{N}$ be the aggregate of all the functionals in $\mathscr{X}^{* *}$ appearing in the expression for $f$, and define a linear operator $T$ from $\mathscr{X}^{*}$ to $\mathbf{C}^{N}$ by

$$
T(z)=\left(\varphi_{1}(z), \ldots, \varphi_{N}(z)\right), \quad z \in \mathscr{X}^{*} .
$$

Let $p$ be the polynomial in the coordinates of $\mathbf{C}^{N}$ associated with $f$, so that

$$
f(z)=p(T(z)), \quad z \in \mathscr{X}^{*} .
$$

Evidently

$$
|p(w)| \leq\|f\|_{B}, \quad w \in T\left(\text { ball } \mathscr{X}^{*}\right)
$$


Let $\left\{T_{\alpha}\right\}$ be a net of linear operators from $\mathscr{X}^{*}$ to $\mathbf{C}^{N}$ as in Lemma 4.2, and define

$$
f_{\alpha}(z)=p\left(T_{\alpha}(z)\right), \quad z \in \mathscr{X}^{*} .
$$

From (4.1) and Lemma 4.2(ii) we obtain $\left|f_{\alpha}(z)\right| \leq\|f\|_{B}$ for all $z \in B$. By Lemma 4.2(i), $f_{\alpha}(z)$ tends to $f(z)$ for all $z \in B$.

Since $T_{\alpha}$ is continuous with respect to the weak-star topology of $\mathscr{X}^{*}$, there are $x_{\alpha}^{1}, \ldots, x_{\alpha}^{N}$ in $\mathscr{X}$ such that

$$
T_{\alpha}(z)=\left(\left\langle z, x_{\alpha}^{1}\right\rangle, \ldots,\left\langle z, x_{\alpha}^{N}\right\rangle\right), \quad z \in \mathscr{X}^{*} .
$$

Then $f_{\alpha}$ is a polynomial in the weak-star continuous functionals on $\mathscr{X}^{*}$ corresponding to the $x_{\alpha}^{j}$ 's.

We wish to give now a concrete criterion for pointwise bounded approximation. Recall that a Banach space $\mathscr{X}$ has the metric approximation property if there is a net $\left\{T_{\alpha}\right\}$ of finite-rank operators on $\mathscr{X}$ such that $\left\|T_{\alpha}\right\| \leq 1$ and $T_{\alpha}(x) \rightarrow x$ for all $x \in \mathscr{X}$. All reasonable Banach spaces, such as $L^{p}$-spaces and the spaces $C(X)$, have the metric approximation property.

4.3. Lemma. Suppose that the Banach space $\mathscr{X}$ has the metric approximation property. Then any bounded analytic function $f$ on ball $\mathscr{X}$ can be approximated there pointwise by a net $\left\{f_{\beta}\right\}$ of (finite) polynomials in elements of $\mathscr{X}^{*}$, satisfying

$$
\left\|f_{\beta}\right\|_{\text {ball } \mathscr{X}} \leq\|f\|_{\text {ball } \mathscr{X}} \text {. }
$$

Proof. Let the finite-rank operators $T_{\alpha}$ be as above. For each $\alpha$, we can find a sequence $\left\{g_{\alpha, m}\right\}$ of analytic polynomials on the range of $T_{\alpha}$, such that $g_{\alpha, m}(x) \rightarrow f(x)$ for all $x$ in the range of $T_{\alpha}$, and such that $\left|g_{\alpha, m}\right|$ is bounded on ball $T_{\alpha}(\mathscr{X})$ by the supremum norm of $f$ on ball $\mathscr{X}$. Then the compositions $f_{\alpha, m}=g_{\alpha, m} \circ T_{\alpha}$ are the desired pointwise approximators to $f$.

The criterion we have been aiming at, guaranteeing that $A_{p b}(B)=H^{\infty}(B)$, now follows immediately upon combining Theorem 4.1 and Lemma 4.3.

4.4. Theorem. Suppose that the dual Banach space $\mathscr{Z}$ has the metric approximation property. Then for every $f \in H^{\infty}(B)$, there is a net $\left\{f_{\alpha}\right\}$ of polynomials in weak-star continuous linear functionals (i.e., $f_{\alpha} \in A(B)$ ) such that

$$
\left\|f_{\alpha}\right\|_{B} \leq\|f\|_{B}, \quad f_{\alpha}(z) \rightarrow f(z), \quad z \in B .
$$

\section{TOPOLOGIES FOR $B$ ASSOCIATED WITH $A(B)$}

We may regard the open unit ball $B$ of $\mathscr{Z}$ as a subset of the dual $A(B)^{*}$ of the uniform algebra $A(B)$, by identifying $z \in B$ with the evaluation functional $\varphi_{z}$ at $z$. As such $B$ inherits three topologies from $A(B)^{*}$ which are of concern to us: the Gleason topology (the relative norm topology from $A(B)^{*}$ ), the weak topology, or $A(B)^{* *}$-topology; and the Gelfand topology (the weak-star toplogy of $\left.A(B)^{*}\right)$. The Gelfand topology of $B$ concides with the relative weak-star 
topology it inherits as a subset of the dual Banach space $\mathscr{Z}$. The Gleason topology of $B$ is identified in the following lemma.

5.1. Lemma. The Gleason topology of $B$ (induced by the norm of $A(B)^{*}$ ) coincides with the norm topology of $B$ (induced by the norm of $\mathscr{Z}$ ).

Proof. The estimate (2.1) yields

$$
\left\|\varphi_{x}-\varphi_{y}\right\|_{A(B)^{*}} \leq 2\|x-y\| /(1-\|x\|), \quad x, y \in B .
$$

On the other hand, the Hahn-Banach theorem provides us with $L \in \mathscr{X}^{*}$ satisfying $\|L\|=1$ and $L(x-y)=\|x-y\|$. The function $F(z)=L(z-y)$ belongs to $A(B)^{* *}$ and satisfies $F(x)=\|x-y\|, F(y)=0$, and $\|F\| \leq 2$. It follows that

$$
\frac{1}{2}\|x-y\| \leq\left\|\varphi_{x}-\varphi_{y}\right\|_{A(B)^{*}},
$$

so that the two topologies are indeed equivalent, with uniform Lipschitz estimates on each fixed subball $r B, 0<r<1$.

In anticipation of our work on tight algebras, we wish now to investigate in more detail the $A(B)^{* *}$-topology of $B$. In view of (3.1), a net $\left\{z_{\alpha}\right\}$ in $B$ converges to $z$ in the $A(B)^{* *}$-topology if and only if $f\left(z_{\alpha}\right) \rightarrow f(z)$ for all $f \in A_{p b}(B)$.

5.2. Lemma. Fix $0<r<1$, and let $\left\{z_{\alpha}\right\}$ be a net in $B$ such that $\left\|z_{\alpha}\right\| \leq r$ for all $\alpha$. Then $z_{\alpha}$ converges to $z$ in the $A(B)^{* *}$-topology if and only if for all $m \geq 1, f\left(z_{\alpha}\right) \rightarrow f(z)$ for every $m$-homogeneous analytic function $f$ in $A_{p b}(B)$.

Proof. This follows from (3.1), and the fact (Lemma 2.1 and Theorem 3.1) that any function in $A_{p b}(B)$ can be approximated uniformly on $r B$ by linear combinations of such $f$ 's.

5.3. Theorem. Suppose that $\mathscr{Z}$ is infinite dimensional. Then for any $0<r<1$, the $A(B)^{* *}$-closure of the sphere $\{\|z\|=r\}$ coincides with the closed ball $r \bar{B}$. In particular, the $A(B)^{* *}$-topology for $B$ is different from the Gleason topology. Proof. It is clear that the closure of the sphere is contained in the ball. Suppose $z_{0} \in B$ satisfies $\left\|z_{0}\right\|<r$. Let $\alpha=\left\{f_{1}, \ldots, f_{k}\right\}$ be any finite collection of homogeneous functions whose restrictions to $B$ belong to $A_{p b}(B)$. For such an $\alpha$ determined by $k$ functions, let $\mathscr{Z}_{\alpha}$ be any subspace of $\mathscr{Z}$ of dimension $k+1$ containing $z_{0}$. Then the set

$$
\left\{f_{1}=f_{1}\left(z_{0}\right)\right\} \cap \cdots \cap\left\{f_{k}=f_{k}\left(z_{0}\right)\right\} \cap \mathscr{Z}_{\alpha}
$$

is a complex analytic variety in $\mathscr{Z}_{\alpha}$ of codimension at least 1 containing $z_{0}$, with no compact branches. By the maximum principle there is then a point $y_{\alpha} \in \mathscr{Z}_{\alpha}$ such that $\left\|y_{\alpha}\right\|=r$ and $y_{\alpha}$ lies in the variety. The $y_{\alpha}$ 's form a net, with the ordering of the indices by inclusion. By construction $f\left(y_{\alpha}\right)=f\left(z_{0}\right)$ eventually, for any $m$-homogeneous function $f$ in $A_{p b}(B)$. By Lemma 5.2, $y_{\alpha}$ converges to $z_{0}$ in the $A(B)^{* *}$-topology. 
The same proof shows that if $\mathscr{Z}$ is any infinite dimensional Banach space and $0<r<1$, then the $H^{\infty}$ (ball $\mathscr{Z}$ )-closure of the sphere $\{\|x\|=r\}$ coincides with $r$ ball $\mathscr{Z}$.

From Theorem 5.3 one can construct a net $\left\{z_{\alpha}\right\}$ in $B$ such that $\left\|z_{\alpha}\right\| \rightarrow$ 1 , while $z_{\alpha}$ converges to 0 in the $A(B)^{* *}$-topology. On the other hand, for sequences a version of the uniform boundedness principle holds.

5.4. Lemma. If $\left\{z_{j}\right\}_{j=1}^{\infty}$ is a sequence in $B$ which converges in the $A(B)^{* *}$ topology, then there is $0<r<1$ such that $\left\|z_{j}\right\| \leq r$ for all $j$.

Proof. Suppose the lemma fails. Passing to a subsequence, we can assume that $\left\|z_{j}\right\| \rightarrow 1$. Then

$$
\lim _{j \rightarrow \infty}\left\|\varphi_{z_{j}}-\varphi_{0}\right\|_{A(B)^{*}}=2,
$$

where $\varphi_{0}$ is the evaluation functional at $0, \varphi_{z_{j}}$ at $z_{j}$. By a general result on uniform algebras, to follow, the $z_{j}$ 's contain an interpolating subsequence for $A(B)^{* *}$, and consequently they cannot converge in the $A(B)^{* *}$-topology.

The general result for function algebras, appealed to above, is as follows.

5.5. Theorem. Let $A$ be a uniform algebra on a compact space $X$, let $x \in X$, and let $\left\{x_{j}\right\}$ be a sequence in $X$ lying in the same Gleason part as $x$, such that

$$
\lim _{j \rightarrow \infty}\left\|\varphi_{x_{j}}-\varphi_{x}\right\|=2,
$$

the norm of the evaluation functionals being that of $A^{*}$. Then the sequence $\left\{x_{j}\right\}$ has an interpolating subsequence for $A^{* *}$.

Proof. Passing to a subsequence, we can assume that $\left\|\varphi_{x_{j}}-\varphi_{x}\right\|$ converges very rapidly to 2 . In view of the relation between the Gleason metric (norm metric of $A^{*}$ ) and Harnack's inequality (cf. [7]), we can find functions $g_{j} \in A$ such that $\operatorname{Re} g_{j}>0, g_{j}(x)=1 / 2^{j}$, and $\operatorname{Re} g_{j}\left(x_{j}\right)>j$. Let $\phi$ be the conformal map of the right half-plane onto the open unit disk such that $\phi(1)=0$ and $\phi(\infty)=1$. Then

$$
f_{m}=\phi\left(g_{1}+\cdots+g_{m}\right)
$$

belongs to $A,\left|f_{m}\right| \leq 1$, and $f_{m}(x) \rightarrow 0$. Let $F \in A^{* *}$ be any weak-star accumulation point of the sequence $\left\{f_{m}\right\}$. Then $|F| \leq 1$, and $F(x)=0$. Since

we have

$$
\operatorname{Re}\left(g_{1}+\cdots+g_{m}\right)\left(x_{j}\right)>j, \quad m \geq j,
$$

$$
f_{m}\left(x_{j}\right) \in \phi(\{\operatorname{Re} \lambda \geq j\}), \quad m \geq j,
$$

and consequently $F\left(x_{j}\right)$ belongs to the closure of the image of the half-plane $\{\operatorname{Re} z \geq j\}$ under $\phi$. It follows that

$$
\lim _{j \rightarrow \infty} F\left(x_{j}\right)=1 .
$$

Note that $\left|F\left(x_{j}\right)\right|<1$, since $x_{j}$ is in the same Gleason part as $x$. Passing to a subsequence, we can assume that $\left\{F\left(x_{j}\right)\right\}$ is an interpolating sequence for the 
algebra $H^{\infty}(\{|\lambda|<1\})$ of bounded analytic functions on the open unit disk. By composing such functions with $F$, we see that $\left\{x_{j}\right\}$ is then interpolating for $A^{* *}$.

In the next section we wish to show that for some specific Banach spaces $\mathscr{Z}$, the sequences in $B$ which converge in the $A(B)^{* *}$-topology are precisely those which converge in norm. Instead of dealing with a dual Banach space, it will be convenient to work with an arbitrary Banach space $\mathscr{X}$ and the algebra $H^{\infty}($ ball $\mathscr{X})$.

\section{6. $\Lambda$-SPACES}

We say that a net $\left\{x_{\alpha}\right\}$ in $\mathscr{X}$ converges in the weak polynomial topology to $x$ if $f\left(x_{\alpha}\right) \rightarrow f(x)$ for all analytic polynomials $f \in \mathscr{P}(\mathscr{X})$. If $\left\{x_{\alpha}\right\}$ converges in norm to $x$, then $\left\{x_{\alpha}\right\}$ is weak polynomial convergent to $x$. In turn, weak polynomial convergence implies weak convergence of a net, since $\mathscr{P}_{1}(\mathscr{X})=$ $\mathscr{X}^{*} \subseteq \mathscr{P}(\mathscr{X})$.

We define a Banach space $\mathscr{X}$ to be a $\Lambda$-space if whenever $\left\{x_{j}\right\}_{j=1}^{\infty}$ is a sequence in $\mathscr{X}$ which is weak polynomial convergent to 0 , then $\left\|x_{j}\right\| \rightarrow 0$.

Recall that a Banach space has the Schur property if whenever $\left\{x_{j}\right\}$ is a sequence in $\mathscr{X}$ which converges weakly to 0 , then $\left\|x_{j}\right\| \rightarrow 0$. Any Banach space with the Schur property is evidently a $\Lambda$-space. Finite dimensional spaces, and the sequence space $l^{1}$, all have the Schur property, so that these spaces are all $\Lambda$-spaces. Since the restriction of any polynomial on $\mathscr{L}$ to a subspace is polynomial, we obtain immediately the following.

6.1. Theorem. Any closed subspace of a $\Lambda$-space is a $\Lambda$-space.

The Banach space $c_{0}$ of null sequences is not a $\Lambda$-space. Indeed, let $\left\{e_{j}\right\}$ be the sequence of standard basis vectors in $c_{0}$. By the Littlewood-Pelczynski Theorem $[10,12]$ any $f \in \mathscr{P}\left(c_{0}\right)$ can be approximated uniformly on the unit ball of $c_{0}$ by finite polynomials in the coordinate functions. It follows that $f\left(e_{j}\right) \rightarrow 0$ for all $f \in \mathscr{P}\left(c_{0}\right)$. Since the $e_{j}$ 's have unit norm, $c_{0}$ fails to be a $\Lambda$-space. Combining this with Theorem 6.1 , we obtain the following useful criterion.

6.2. Theorem. If the Banach space $\mathscr{X}$ has a (closed) subspace isomorphic to $c_{0}$, then $\mathscr{Z}$ is not a $\Lambda$-space.

From this criterion, it follows for instance that no infinite dimensional uniform algebra can be a $\Lambda$-space. Indeed, if $A$ is an infinite dimensional uniform algebra, and if $\varepsilon>0$, then there is a linear isomorphism $T$ of $c_{0}$ into $A$ such that

$$
(1-\varepsilon)\|T x\| \leq\|x\| \leq(1+\varepsilon)\|T x\|, \quad x \in c_{0} .
$$

The proof of this well-known fact runs along the following lines. Let $\left\{U_{j}\right\}$ be a sequence of nonempty disjoint open subsets of the Shilov boundary of $A$. 
Choose $g_{j} \in A$ such that $\left\|g_{j}\right\|=1$, and $\left|g_{j}\right|<\varepsilon / 2^{j+1}$ off $U_{j}$ for $j \geq 1$. Define $T$ on $c_{0}$ by

$$
T x=\sum x_{j} g_{j}, \quad x \in c_{0} .
$$

One checks easily that $T$ has the required properties.

Our aim in the remainder of this section and the next is to establish the following assertions:

$$
\begin{gathered}
l^{p} \text { is a } \Lambda \text {-space for } 1 \leq p<\infty . \\
L^{p}(\mu) \text { is a } \Lambda \text {-space for } 2 \leq p<\infty . \\
L^{1}[0,1] \text { is not a } \Lambda \text {-space. }
\end{gathered}
$$

We do not know whether $L^{p}(\mu)$ is a $\Lambda$-space, for $1<p<2$.

The following theorem is a sharp version of (6.1).

6.3. Theorem. Let $1 \leq p<\infty$, and let $N$ be an integer satisfying $N \geq p$. Suppose $\left\{f_{j}\right\}_{j=1}^{\infty}$ is a sequence in $l^{p}$ such that $f_{j} \rightarrow 0$ weakly, and $F\left(f_{j}\right) \rightarrow 0$ for every $f \in \mathscr{P}_{N}\left(l^{p}\right)$. Then $\left\|f_{j}\right\|_{p} \rightarrow 0$.

Proof. The $f_{j}$ 's are bounded, so we assume that $\left\|f_{j}\right\|_{p} \leq 1$ for all $j$. Note that for purposes of proving that $f_{j} \rightarrow 0$ in norm, we can pass to subsequences whenever convenient. We can also replace $f_{j}$ by any sequence $g_{j}$ which is asymptotic to $f_{j}$, in the sense that $\left\|f_{j}-g_{j}\right\|_{p} \rightarrow 0$.

Since $f_{j} \rightarrow 0$ weakly, also $f_{j} \rightarrow 0$ pointwise. We can then, by passing to a subsequence, assume that there are blocks $E_{j}=\left\{m_{j} \leq k \leq n_{j}\right\}$ of integers so that the blocks $E_{j}$ are pairwise disjoint, and so that $\left\|f_{j} \chi_{E_{j}}-f_{j}\right\|_{p}<1 / 2^{j}$. Replacing $f_{j}$ by $f_{j} \chi_{E_{j}}$, we can assume then that $f_{j}=0$ off $E_{j}$. Now define the $N$-homogeneous function $F$ on $l^{p}$ by

$$
F(f)=\sum\left\langle f, \bar{f}_{j}\left|f_{j}\right|^{p-2}\right\rangle^{N},
$$

where

$$
\langle f, g\rangle=\sum_{k=1}^{\infty} f(k) g(k) .
$$

Let $q=p /(p-1)$ be the conjugate index to $p$. Since the $l^{p}$-norms of the $f_{j}$ 's are bounded by 1 , the $l^{q}$-norms of $\bar{f}_{j}\left|f_{j}\right|^{p-2}$ are also bounded by 1 . Suppose $f \in l^{p}$ satisfies $\|f\|_{p} \leq 1$. Then using Hölder's inequality we obtain

$$
\begin{aligned}
& \sum\left|\left\langle f, \bar{f}_{j}\left|f_{j}\right|^{p-2}\right\rangle\right|^{N} \leq \sum_{j}\left\|f \chi_{E_{j}}\right\|_{p}^{N} \\
& =\sum_{j}\left[\sum_{k \in E_{j}}|f(k)|^{p}\right]^{N / p} \leq \sum_{j} \sum_{k \in E_{j}}|f(k)|^{p} \leq 1 .
\end{aligned}
$$


This shows that the series defining $F(f)$ converges absolutely, and that $F$ is bounded by 1 on the unit ball of $l^{p}$. By hypothesis, $F\left(f_{j}\right) \rightarrow 0$. Since

$$
F\left(f_{j}\right)=\left\langle f_{j}, \bar{f}_{j}\left|f_{j}\right|^{p-2}\right\rangle^{N}=\left\|f_{j}\right\|_{p}^{p N},
$$

we obtain $\left\|f_{j}\right\|_{p} \rightarrow 0$, as required.

Note that the condition $N \geq p$ in Theorem 6.3 is sharp. This follows from Pitt's theorem [14], which asserts that if $1 \leq N<p$, then every continuous $N$ linear form in $l^{p}$ can be approximated uniformly by $N$-linear forms depending on only a finite number of coordinates. Indeed, suppose $1<p<\infty$, and let $\left\{e_{j}\right\}$ be the sequence of basis vectors in $l^{p}$, so that $\left\|e_{j}\right\|=1$ while $e_{j} \rightarrow 0$ weakly. If $N<p$, then by Pitt's theorem $F\left(e_{j}\right) \rightarrow 0$ for every $F \in \mathscr{P}_{N}$, and Theorem 6.3 fails for $N<p$.

Next we consider spaces of the form $L^{p}(\mu)$. Since Theorem 6.3 is valid for $l^{2}$, it is valid for any Hilbert space, hence for $L^{2}(\mu)$. For other $L^{p}$-spaces we begin with the following sharper version of (6.2).

6.4. Theorem. Let $\mu$ be a positive measure on some measure space, let $2 \leq$ $p<\infty$, and let $N$ be an integer satisfying $N \geq p$. Let $\left\{f_{j}\right\}$ be a sequence in $L^{p}(\mu)$ such that $f_{j} \rightarrow 0$ weakly, and $F\left(f_{j}\right) \rightarrow 0$ for every $F \in \mathscr{P}_{N}\left(L^{p}(\mu)\right)$. Then $\left\|f_{j}\right\|_{p} \rightarrow 0$.

Proof. Since the $f_{j}$ 's are all carried by some $\sigma$-finite set, we can assume that the measure $\mu$ is $\sigma$-finite. Replacing $\mu$ by a mutually absolutely continuous measure, we can assume that $\mu$ is a probability measure. We further assume that the $f_{j}$ 's are bounded by 1 in the norm of $L^{p}(\mu)$. Then they are also bounded by 1 in the norm of $L^{2}(\mu)$, and $f_{j} \rightarrow 0$ weakly in $L^{2}(\mu)$.

If $G$ is an $N$-homogeneous analytic function bounded on ball $L^{2}(\mu)$, then $G$ restricts to an $N$-homogeneous analytic function bounded on ball $L^{p}(\mu)$. Consequently $G\left(f_{j}\right) \rightarrow 0$. By the version of the result already proved for Hilbert space, we obtain $\left\|f_{j}\right\|_{2} \rightarrow 0$. Passing to a subsequence, we can assume that $f_{j}$ converges almost uniformly to 0 .

Now we proceed as in the proof for the $l^{p}$-spaces. Passing to a subsequence, we construct by induction pairwise disjoint measurable sets $E_{j}$ such that $\left\|f_{j} \chi_{E_{j}}-f_{j}\right\|_{p} \rightarrow 0$. Replacing $f_{j}$ by $f_{j} \chi_{E_{j}}$, we can then assume that $f_{j}$ is carried by $E_{j}$. Now define

$$
\begin{aligned}
F(f) & =\sum\left\langle f, \bar{f}_{j}\left|f_{j}\right|^{p-2}\right\rangle^{N} \\
& =\sum\left[\int_{E_{j}} f(t) \overline{f_{j}(t)}\left|f_{j}(t)\right|^{p-2} d \mu(t)\right]^{N} .
\end{aligned}
$$

As before the series converges absolutely to an $F \in \mathscr{P}_{N}$. By hypothesis, $F\left(f_{j}\right) \rightarrow$ 0 . Since $F\left(f_{j}\right)=\left\|f_{j}\right\|_{p}^{p N}$, we obtain $\left\|f_{j}\right\|_{p} \rightarrow 0$, as required. 
If $L^{p}(\mu)$ is infinite dimensional, the condition $N \geq p$ is sharp. This can be seen by embedding $l^{p}$ isometrically into $L^{p}(\mu)$ and applying Pitt's theorem as before.

Next we aim to verify $(6.3)$, that $L^{1}[0,1]$ is not a $\Lambda$-space. We will actually establish the following.

6.5. Theorem. The following are equivalent, for a measure $\mu$ :

(i) $L^{1}(\mu)$ is a $\Lambda$-space.

(ii) $L^{1}(\mu)$ has the Schur property.

(iii) $\mu$ is atomic.

Proof. Schur's theorem asserts that $l^{1}$ has the Schur property, and the straightforward proof shows that $L^{1}(\mu)$ has the Schur property whenever $\mu$ is atomic. On the other hand, in the case $\mu$ is not atomic it is easy to construct a sequence in $L^{1}(\mu)$ which converges weakly to 0 but not strongly. In fact, any such $L^{1}(\mu)$ contains an isometric copy of $L^{1}[0,1]$, where the functions $\sin (2 \pi m t)$ converge weakly but not strongly to 0 as $m \rightarrow \infty$.

If a Banach space has the Schur property, then trivially it is a $\Lambda$-space. On the other hand, using the representation of the projective tensor product of $L^{1}$-spaces as an $L^{1}$-space of the corresponding product measure, it is a straightforward matter to check that if a sequence in $L^{1}(\mu)$ tends to zero weakly, then it is in fact a weak polynomial null sequence. Thus if the Schur property fails in $L^{1}(\mu)$, then $L^{1}(\mu)$ is not a $\Lambda$-space.

Rather than give more details here, we will prove in the next section a general result for spaces with the Dunford-Pettis property, which yields as a corollary the equivalence of weak and weak polynomial convergence of sequences in $L^{1}(\mu)$.

\section{Relation to the Dunford-Pettis PROPERTY}

Recall that the Banach space $\mathscr{X}$ has the Dunford-Pettis property if whenever $\left\{x_{j}\right\}$ is a weak null sequence in $\mathscr{X}$ and $\left\{\varphi_{j}\right\}$ is a weak null sequence in $\mathscr{X}^{*}$, then $\varphi_{j}\left(x_{j}\right) \rightarrow 0$. For a discussion of the Dunford-Pettis property, see [6]. Spaces which enjoy the Dunford-Pettis property are $C(X)$ and $L^{1}(\mu)$. Our main aim in this section is to establish the following theorem.

7.1. Theorem. Suppose that $\mathscr{Z}$ has the Dunford-Pettis property. Then the restriction of any polynomial on $\mathscr{Z}$ to a weakly compact set is weakly continuous. In particular, the weak topology coincides with the weak polynomial topology on weakly compact sets.

For the proof, we begin with the following simple consequence of the Eberlein-Schmulian theorem.

7.2. Lemma. Let $E$ be a weakly compact subset of a Banach space $\mathscr{Z}$, and let $h$ be a function from $E$ to a Banach space $\mathscr{Y}$. Suppose that $h$ is sequentially 
continuous with respect to the weak topologies of $\mathscr{X}$ and $\mathscr{Y}$, that is, if $\left\{x_{n}\right\}$ is a sequence in $E$ converging weakly to $x$, then $h\left(x_{n}\right)$ converges weakly in $\mathscr{y}$ to $h(x)$. Then $h$ is continuous with respect to the weak topologies.

Proof. It suffices to show that the graph $\Gamma$ of $h$ is weakly compact in $\mathscr{X} \oplus \mathscr{Y}$.

Let $\left\{\left(x_{n}, h\left(x_{n}\right)\right)\right\}$ be a sequence in $\Gamma$. Since $E$ is weakly compact, there is by the Eberlein-Schmulian theorem a subsequence $\left\{x_{n_{j}}\right\}$ which converges weakly to $x \in E$. By the assumption on $h, h\left(x_{n_{j}}\right)$ converges weakly in $\mathscr{Y}$ to $h(x)$. Hence $\left\{\left(x_{n_{j}}, h\left(x_{n_{j}}\right)\right\}\right.$ converges weakly in $\mathscr{X} \oplus \mathscr{Y}$ to $(x, h(x)) \in \Gamma$, and $\Gamma$ is weakly sequentially compact in $\mathscr{X} \oplus \mathscr{Y}$. By the Eberlein-Schmulian theorem, $\Gamma$ is weakly compact.

Note that the preceding lemma shows in particular that a scalar-valued function on the weakly compact set $E$ is weakly continuous if and only if it is weakly sequentially continuous.

7.3. Lemma. The following are equivalent, for a Banach space $\mathscr{X}$.

(i) Each weakly compact subset of $\mathscr{X}$ is weakly polynomially compact.

(ii) The restriction of any polynomial to a weakly compact subset of $\mathscr{X}$ is weakly continuous.

(iii) Any polynomial on $\mathscr{X}$ is weakly sequentially continuous.

(iv) If $\left\{x_{k}\right\}_{k=1}^{\infty}$ is a weak null sequence in $\mathscr{X}$, then for any $m \geq 1,\left\{x_{k} \otimes \cdots \otimes\right.$ $\left.x_{k}\right\}$ is a weak null sequence in the projective tensor product $\mathscr{X} \widehat{\otimes} \cdots \widehat{\otimes} \mathscr{X}$ ( $m$ times).

(v) For any $m \geq 1$, the function

$$
\theta: \mathscr{X} \rightarrow \mathscr{X} \widehat{\otimes} \cdots \widehat{\otimes} \mathscr{X}(m \text { times })
$$

defined by $\theta(x)=x \otimes \cdots \otimes x$ is sequentially continuous, from the weak topology of $\mathscr{X}$ to the weak topology of $\mathscr{X} \widehat{\otimes} \cdots \widehat{\otimes} \mathscr{X}$.

(vi) The restriction of $\theta$ to any weakly compact subset of $\mathscr{X}$ is continuous with respect to the weak topologies of $\mathscr{X}$ and of $\mathscr{X} \widehat{\otimes} \cdots \widehat{\otimes} \mathscr{X}$.

Proof. The equivalence of (i) and (ii) is clear. That (iii) implies (ii) follows from Lemma 7.2, so that (ii) and (iii) are also equivalent. The equivalence of (v) and (vi) also follows from Lemma 7.2.

Suppose that (v) holds, and let $f$ be an $m$-homogeneous analytic function on $\mathscr{X}$. Then $f$ is the restriction to the diagonal of a continuous $m$-linear form $F$ on $\mathscr{X}$. The space of such forms is equivalent to the dual of the $m$ fold projective tensor product $\mathscr{X} \widehat{\otimes} \cdots \widehat{\otimes} \mathscr{X}$, via the correspondence $F \leftrightarrow L$, where $L\left(x_{1} \otimes \cdots \otimes x_{m}\right)=F\left(x_{1}, \ldots, x_{m}\right)$. Now $f$ is the composition $L \circ \theta$ of the weakly sequentially continuous functions $L$ and $\theta$. It follows that $f$ is weakly sequentially continuous, and (iii) is valid.

Suppose next that (iii) holds. Suppose $\left\{x_{k}\right\}$ is a sequence in $\mathscr{X}$ converging weakly to $x$. Let $L \in(\mathscr{X} \widehat{\otimes} \cdots \widehat{\otimes} \mathscr{X})^{*}$, and let $F$ be the corresponding continuous $m$-linear form on $\mathscr{X}$. By (iii), $F\left(x_{k}, \ldots, x_{k}\right)=L\left(\theta\left(x_{k}\right)\right)$ converges 
to $F(x, \ldots, x)=L(\theta(x))$. Thus $\theta\left(x_{k}\right)$ converges weakly to $\theta(x)$, and $(\mathrm{v})$ is valid.

Since (v) implies (iv) trivially, it remains only to show that (iv) implies (v).

Suppose (iv) holds, and fix $m \geq 1$. Let $\left\{x_{k}\right\}$ be a sequence in $\mathscr{X}$ which converges weakly to $x$. Let $L \in(\mathscr{X} \hat{\otimes} \cdots \widehat{\otimes} \mathscr{X})^{*}$, and let $F$ be the corresponding $m$-linear form, as above. Write $x_{k}=x+y_{k}$, where $\left\{y_{k}\right\}$ is a weak null sequence. Then

$$
\begin{aligned}
L\left(\theta\left(x_{k}\right)\right) & =F\left(x_{k}, \ldots, x_{k}\right)=F\left(x+y_{k}, \ldots, x+y_{k}\right) \\
& =F(x, \ldots, x)+F\left(x, \ldots, x, y_{k}\right)+\cdots+F\left(y_{k}, \ldots, y_{k}\right) .
\end{aligned}
$$

Invoking the hypothesis (iv) for the $q$-fold projective tensor products of $\mathscr{X}$ with itself, for $1 \leq q \leq m$, and noting that $x$ is fixed, we see that each of the summands involving the $y_{k}$ 's tends to 0 . Hence $L\left(\theta\left(x_{k}\right)\right) \rightarrow F(x, \ldots, x)=$ $L(\theta(x))$, and $(v)$ holds.

To prove Theorem 7.1, it suffices now to verify the condition (iv) of Lemma 7.3 in the case $\mathscr{Z}$ has the Dunford-Pettis property. In fact, the following is true.

7.4. Lemma. Let $m \geq 1$, and let $\mathscr{X}_{1}, \ldots, \mathscr{X}_{m}$ be Banach spaces, of which all but at most one have the Dunford-Pettis property. Let $\left\{x_{j, k}\right\}_{k=1}^{\infty}$ be a weak null sequence in $\mathscr{X}_{j}, 1 \leq j \leq m$. Then $\left\{x_{1, k} \otimes \cdots \otimes x_{m, k}\right\}_{k=1}^{\infty}$ is a weak null sequence in the projective tensor product $\mathscr{X}_{1} \widehat{\otimes} \cdots \widehat{\otimes}_{m}$.

Proof. The lemma is trivial if $m=1$. Suppose that $m \geq 2$, and that $\mathscr{L}_{2}, \ldots$, $\mathscr{X}_{m}$ have the Dunford-Pettis property. Set $\mathscr{W}=\mathscr{X}_{1} \widehat{\otimes} \cdots \widehat{\otimes}_{m-1}$. By an induction hypothesis we may assume that the sequence $w_{k}=x_{1, k} \otimes \cdots \otimes x_{m-1, k}$ is a weak null sequence in $\mathscr{W}$. Let $F \in\left(\mathscr{X}_{1} \widehat{\otimes} \cdots \widehat{\otimes}_{m}\right)^{*}=\left(\mathscr{W} \widehat{\otimes} \mathscr{X}_{m}\right)^{*}$. This dual space is isometric to the space of bilinear forms on $\mathscr{W} \times \mathscr{X}_{m}$. Now the bilinear forms on $\mathscr{W} \times \mathscr{Z}_{m}$ correspond to operators $T: \mathscr{W} \rightarrow \mathscr{X}_{m}^{*}$ in such a way that $F$ and $T$ are related by

$$
F(w \otimes x)=\langle T(w), x\rangle, \quad w \in \mathscr{W}, x \in \mathscr{X}_{m} .
$$

Now $T\left(w_{k}\right)$ is also a weak null sequence in $\mathscr{Z}_{m}^{*}$. Since $\mathscr{Z}_{m}$ has the DunfordPettis property, we obtain

$$
F\left(x_{1 k} \otimes \cdots \otimes x_{m k}\right)=\left\langle T\left(w_{k}\right), x_{m, k}\right\rangle \rightarrow 0 .
$$

This establishes the lemma, and the proof of Theorem 7.1 is thereby complete.

We remark that on the basis of Lemma 7.4, a stronger property than Lemma 7.3(vi) can be established for spaces with the Dunford-Pettis property. Indeed the same proof shows that the condition in Lemma 7.4 implies that the map

$$
\left(x_{1}, \ldots, x_{m}\right) \rightarrow x_{1} \otimes \cdots \otimes x_{m}
$$

is weakly continuous from any weakly compact subset of $\mathscr{X}_{1} \oplus \cdots \oplus \mathscr{X}_{m}$ to the projective tensor product $\mathscr{X}_{1} \widehat{\otimes} \cdots \widehat{\otimes} X_{m}$, equipped with the weak topology. 
7.5. Theorem. A Banach space $\mathscr{X}$ has the Schur property if and only if $\mathscr{X}$ is a $\Lambda$-space and $\mathscr{Z}$ has the Dunford-Pettis property.

Proof. We have already observed that any Banach space with the Schur property is a $\Lambda$-space, and it is likewise trivial that the Schur property implies the Dunford-Pettis property. For the reverse implication, suppose that $\mathscr{X}$ is a $\Lambda$ space with the Dunford-Pettis property. Let $\left\{x_{k}\right\}$ be a weak null sequence in $\mathscr{X}$. By Theorem 7.1, $\left\{x_{k}\right\}$ is a weak polynomial null sequence. By the definition of $\Lambda$-space, $x_{k}$ converges to zero is norm, and consequently $\mathscr{X}$ has the Schur property.

As remarked before, from Theorem 7.4 one sees that $C(X)$ and $L^{1}(\mu)$ are not $\Lambda$-spaces, when $X$ is an infinite compact space and the measure $\mu$ is not atomic. Indeed, these spaces have the Dunford-Pettis property, while the Schur property is easily seen to fail.

\section{Tight UNIFORM ALGEBRAS}

In this section, we prove some preliminary lemmas on tight algebras and compactly tight algebras, in the context of an arbitrary uniform algebra $A$ on a compact space $X$. The lemmas represent sharpened versions of some results in [2], designed to treat the algebras at hand. by

For $g \in C(X)$ the Hankel-type operator $S_{g}$ from $A$ to $C(X) / A$ is defined

$$
S_{g}(f)=g f+A, \quad f \in A .
$$

The algebra $A$ is tight on $X$ if the operators $S_{g}$ are weakly compact, for all $g \in C(X)$. The algebra $A$ is compactly tight if each $S_{g}$ is a compact operator. We begin with the following.

8.1. Lemma. Let $A$ be a uniform algebra in a compact space $X$. Let $g \in C(X)$, and let $\left\{\nu_{\alpha}\right\}$ be a bounded net of measures on $X$ such that $\nu_{\alpha} \perp A$ for all $\alpha$, and $\nu_{\alpha}$ converges weak-star to 0 . If the operator $S_{g}$ is weakly compact, then

$$
\lim _{\alpha} \int F g d \nu_{\alpha}=0
$$

for all $F \in A^{* *}$, that is, $g \nu_{\alpha}$ converges weakly to 0 in $A^{*}$. If $S_{g}$ is compact, then the convergence is uniform over $F \in \operatorname{ball}\left(A^{* *}\right)$, that is $g \nu_{\alpha}$ converges to 0 in the norm of $A^{*}$.

Proof. The adjoint of $S_{g}$ is the operator

$$
S_{g}^{*}: A^{\perp} \rightarrow A^{*}
$$

given by the pairing

$$
\left\langle S_{g}^{*}(\nu), f\right\rangle=\int f g d \nu, \quad f \in A .
$$


If $S_{g}$ is weakly compact, so is $S_{g}^{*}$, and the net $\left\{g \nu_{\alpha}\right\}$ is a weakly precompact subset of $A^{*}$. Since $\nu_{\alpha} \rightarrow 0$ weak-star, the only weak limit point of the net $\left\{g \nu_{\alpha}\right\}$ in $A^{*}$ is 0 , and consequently $\left\{g \nu_{\alpha}\right\}$ converges weakly to 0 in $A^{*}$.

If $S_{g}$ is compact, then so is $S_{g}^{*}$, and $\left\{g \nu_{\alpha}\right\}$ is a norm compact subset of $A^{*}$. In this case we conclude that $\left\{g \nu_{\alpha}\right\}$ converges to 0 in the norm of $A^{*}$.

8.2. Lemma. Let $A$ be a uniform algebra on $X$, and let $\left\{x_{\alpha}\right\}$ be a net in $X$ converging to $x_{0} \in X$. Let $\mu_{\alpha}$ be a representing measure on $X$ for $x_{\alpha}$ such that the net $\left\{\mu_{\alpha}\right\}$ converges weak-star to a measure $\mu$ satisfying $\mu\left(\left\{x_{0}\right\}\right)=0$. If $A$ is tight on $X$, then

$$
\lim _{\alpha} F\left(x_{\alpha}\right)=F\left(x_{0}\right), \quad \text { all } F \in A^{* *},
$$

that is, $\left\{x_{\alpha}\right\}$ converges weakly to $x_{0}$ in $A^{*}$. If $A$ is compactly tight on $X$, then the convergence is uniform over $F \in$ ball $A^{* *}$, that is, $\left\{x_{\alpha}\right\}$ converges to $x_{0}$ in the norm of $A^{*}$.

Proof. Let $\delta_{\alpha}$ be the point mass at $x_{\alpha}, \delta_{0}$ at $x_{0}$. Then $\nu_{\alpha}=\mu_{\alpha}-\delta_{\alpha}$ converges weak-star to $\nu=\mu-\delta_{0}$. Let $U$ be a neighborhood of $x_{0}$ such that $\mu(U)<\varepsilon$. Shrinking $U$, we can assume that $\mu_{\alpha}(U)<\varepsilon$ for $\alpha$ large, choose $g \in C(X)$ so that $0 \leq g \leq 1, g=0$ on $X \backslash U$, and $g=1$ in a neighborhood of $x_{0}$. By Lemma 8.1,

$$
\lim _{\alpha} \int F g d\left(\nu_{\alpha}-\nu\right)=0, \quad F \in A^{* *},
$$

so that

$$
\lim _{\alpha}\left[F\left(x_{0}\right)-F\left(x_{\alpha}\right)+\int g F d \mu_{\alpha}-\int g F d \mu\right]=0, \quad F \in A^{* *} .
$$

Since $\left\|g \mu_{\alpha}\right\| \leq \varepsilon$ and $\|g \mu\|<\varepsilon$, we obtain

$$
\lim \sup \left|F\left(x_{\alpha}\right)-F\left(x_{0}\right)\right| \leq \lim \sup \left|\int g F d \mu_{\alpha}-\int g F d \mu\right| \leq 2 \varepsilon\|F\| .
$$

This gives the desired pointwise convergence, with uniform convergence over ball $A^{* *}$ when the convergence in (8.1) is uniform.

An analytic disk in $X$ will be for us a nonconstant map $\varphi$ of the open unit disk $\Delta$ into $X$ such that $f \circ \varphi$ is analytic on $\Delta$ for all $f \in A$. Then also $F \circ \varphi$ is analytic for all $F \in A^{* *}$. Moreover, $\varphi(\Delta)$ is contained in a single Gleason part of $A$. As an application of the preceding lemma we give the following criterion for tightness, based on analytic disks.

8.3. Lemma. Suppose $A$ is tight on $X$. Let $\rho_{\alpha}: \Delta \rightarrow X$ be analytic disks, and suppose $\rho_{\alpha}(0)=x_{\alpha}$ converges to $x_{0}$. Suppose also that for some $f \in A$ the net $f \circ \rho_{\alpha}$ converges to a nonconstant analytic function on $\Delta$. Then $F\left(x_{\alpha}\right) \rightarrow F(x)$ for all $F \in A^{* *}$. If moreover $A$ is compactly tight, then this convergence is uniform over all $F \in$ ball $A^{* *}$.

Proof. Fix $r<1$, and let $\sigma_{r}$ be the normalized arc-length measure $d \theta / 2 \pi$ on the circle $\{|z|=r\}$ in $\Delta$. Then $\nu_{\alpha}=\rho_{\alpha}^{*} \sigma_{r}$ is a representing measure on $X$ 
for $x_{0}$. The $\rho_{\alpha}$ 's are locally equicontinuous. Let $\rho$ be a weak limit of the $\rho_{\alpha}$ 's and let $\mu$ be a weak-star cluster point of the $\nu_{\alpha}$ 's. Then $\mu=\rho^{*} \sigma_{r}$. Take $f \in A$ such that $f \circ \rho_{\alpha}$ does not converge to a constant. Then for large $\alpha$, $f \circ \rho_{\alpha} \neq\left(f \circ \rho_{\alpha}\right)(0)$ a.e. $d \sigma_{r}$, so that $f \neq f\left(x_{\alpha}\right)$ a.e. $d \mu$, and $\mu\left(\left\{x_{0}\right\}\right)=0$. Now we can apply Lemma 8.2.

Something can be recovered from the proof of Lemma 8.2 when the limit measure $\mu$ is allowed to have some mass at $x_{0}$, as long as $\mu \neq \delta_{0}$. We will use $M_{A}$ to denote the spectrum of $A$, and $M_{A}$... to denote the spectrum of the bidual algebra $A^{* *}$.

8.4. Lemma. Suppose that $A$ is a tight uniform algebra in $X$. Let $\left\{x_{\alpha}\right\}$ be a net in $X$ converging to $x_{0} \in X$, and let $\mu_{\alpha}$ be a representing measure on $X$ for $x_{\alpha}$ such that $\left\{\mu_{\alpha}\right\}$ converges weak-star to $\mu$. Suppose that $\mu\left(\left\{x_{0}\right\}\right)=c$, where $0 \leq c<1$. Then the $x_{\alpha}$ 's eventually lie in the same Gleason part of $M_{A}$ as $x_{0}$. Any weak-star adherent point $\varphi$ in $M_{A^{*}}$ of the net $\left\{x_{\alpha}\right\}$, regarded as weak-star continuous homomorphisms in $M_{A^{*}}$, belongs to the same Gleason part of $M_{A^{* *}}$ as $x_{0}$ and satisfies $\left\|\varphi-x_{0}\right\| \leq 2 c$, the norm being that of the dual space of $A^{* * *}$. Furthermore, if $A$ is compactly tight, then

$$
\lim _{\alpha} \sup \left\|x_{\alpha}-x_{0}\right\| \leq 2 c .
$$

Proof. The first statement follows from [2, Theorem 10.2], that the operator $\lambda \rightarrow F \lambda, \lambda \in A^{\perp}$, is weak-star to weak-star continuous for each fixed $F \in A^{* *}$. If we apply this to the idempotent $P \in A^{* *}$ corresponding to the Gleason part of $x_{0}$, we see that $P\left(\mu_{\alpha}-\delta_{\alpha}\right)$ converges a weak-star to $P\left(\mu-\delta_{0}\right)$. Now $P\left(\mu-\delta_{0}\right)=\mu-\delta_{0}$ is nonzero by hypothesis, while $P\left(\mu_{\alpha}-\delta_{\alpha}\right)$ is $\mu_{\alpha}-\delta_{\alpha}$ or 0 depending respectively on whether $x_{\alpha}$ is in the same Gleason part as $x_{0}$ or not. From this the first assertion follows.

For the second statement, choose $U$ and $g$ as in the proof of Lemma 8.2, so that $\mu(U)<c+\varepsilon$ and $\mu_{\alpha}(U)<c+\varepsilon$. The estimate (8.1) then yields

$$
\lim \sup \left|F\left(x_{\alpha}\right)-F\left(x_{0}\right)\right| \leq 2(c+\varepsilon)\|F\|, \quad F \in A^{* *} .
$$

Since $F(\varphi)$ is a cluster point of $\left\{F\left(x_{\alpha}\right)\right\}$, we obtain

$$
\left|F(\varphi)-F\left(x_{0}\right)\right| \leq 2 c\|F\|, \quad F \in A^{* *} .
$$

This yields the norm estimate for $\varphi-x_{0}$, and it shows that $\varphi$ and $x_{0}$ are in the same Gleason part of $M_{A} \ldots$.

Suppose now that $A$ is compactly tight. By Lemma 8.1, the norm of $g\left(\mu_{\alpha}-\delta_{\alpha}-\mu+\delta_{0}\right)$ in $A^{*}$ tends to 0 . Since $g\left(x_{\alpha}\right)=1$ for $\alpha$ large, we obtain for $\alpha$ large that

$$
\sup _{F \in \text { ball } A^{. *}}\left|\int F g d \mu_{\alpha}-\int F g d \mu-F\left(x_{\alpha}\right)+F\left(x_{0}\right)\right|<\varepsilon .
$$

Now both $\int F g d \mu_{\alpha}$ and $\int F g d \mu$ are bounded in modulus by $\|F\| \mu_{\alpha}(U) \leq$ $(c+\varepsilon)\|F\|$. From this we obtain

$$
\left|F\left(x_{\alpha}\right)-F\left(x_{0}\right)\right| \leq \varepsilon+2(c+\varepsilon)\|F\|
$$


for $\alpha$ large. Hence

$$
\left\|x_{\alpha}-x_{0}\right\| \leq 2 c+3 \varepsilon
$$

for $\alpha$ large, and we obtain the final statement of the lemma.

A standard example from rational approximation theory (the roadrunner set) shows that we cannot expect $x_{\alpha}$ to converge to $x_{0}$ in Lemma 8.4.

\section{WHEN IS $A(B)$ TIGHT?}

Now we return to the uniform algebra $A(B)$ generated by the weak-star continuous linear functionals on the closed unit ball $\bar{B}$ of the dual Banach space $\mathscr{Z}$. The preceding lemmas give us some information on $\mathscr{Z}$ in the case in which $A(B)$ is tight on $\bar{B}$.

9.1. Theorem. If $A(B)$ is tight on $\bar{B}$, then $\mathscr{Z}$ is reflexive. Furthermore for each $0<r<1$ the $A(B)^{* *}$-topology on $r B$ coincides with the weak-star topology on $r B$.

Proof. Let $\left\{x_{\alpha}\right\}$ be a net in $r B$ which converges weak-star to $x$. Fix $z \in \mathscr{Z}$ such that $0<\|z\|<1-r$. Then the maps

$$
\rho_{\alpha}(\lambda)=x_{\alpha}+\lambda z, \quad|\lambda| \leq 1,
$$

are analytic disks in $B$, which converge weak-star to

$$
\rho(\lambda)=x+\lambda z, \quad|\lambda| \leq 1 .
$$

If $L$ is any weak-star continuous linear functional on $\mathscr{Z}$ for which $L(z) \neq 0$, then $L \circ \rho$ is nonconstant. By Lemma 8.3, $\left\{x_{\alpha}\right\}$ converges to $x$ in the $A(B)^{* *}$ topology. Hence the $A(B)^{* *}$-topology coincides with the weak-star topology on $r B$. Since the weak topology on $r B$ is between the weak-star and the $A(B)^{* *}$-topologies, the weak-star and weak topologies coincide on $r B$. Thus by Alaoglu's theorem closed balls in $B$ are weakly compact, and consequently $\mathscr{Z}$ is reflexive.

9.2. Theorem. If $A(B)$ is compactly tight, then $\mathscr{Z}$ is finite dimensional.

Proof. Let $\left\{x_{\alpha}\right\}$ be as above. Then $F\left(x_{\alpha}\right) \rightarrow F(x)$ uniformly over bounded subsets of $A(B)^{* *}$, hence uniformly for all $F \in \mathscr{Z}^{*}$. Hence $\left\|x_{\alpha}-x\right\| \rightarrow 0$, and the weak-star topology coincides with the norm topology on $r B$. It follows that $\bar{B}$ is norm compact, so $\mathscr{Z}$ is finite dimensional.

The following criterion covers the $\ell^{p}$ spaces for $1 \leq p<\infty$.

9.3. Theorem. Suppose that the dual Banach space $\mathscr{Z}$ is a $\Lambda$-space, and that $\mathscr{Z}$ has the metric approximation property. If $A(B)$ is tight, then $\mathscr{Z}$ is finite dimensional.

Proof. Suppose that $\mathscr{Z}$ is infinite dimensional. By the Josefson-Nissenzweig theorem (see [5]), there is then a sequence $\left\{z_{j}\right\}_{j=1}^{\infty}$ in $\mathscr{Z}$ such that $\left\|z_{j}\right\|=$ $1 / 2, j \geq 1$, and $z_{j}$ converges weak-star to 0 . By Theorem 9.1, $z_{j}$ converges 
to 0 in the $A(B)^{* *}$-topology. By Theorem 3.1, $A(B)$ is pointwise boundedly dense in $H^{\infty}(B)$, so that $f\left(z_{j}\right) \rightarrow 0$ for all $f \in H^{\infty}(B)$. Since $\mathscr{Z}$ is a $\Lambda$-space, $\left\|z_{j}\right\| \rightarrow 0$. This contradiction establishes the theorem.

The following criterion covers the spaces of the form $L^{p}(\mu), 1<p<\infty$.

9.4. Theorem. Suppose that for some $1<p<\infty$, the dual Banach space $\mathscr{Z}$ has a quotient space isomorphic to $\ell^{p}$. Then $A(B)$ is not tight.

Proof. In view of Theorem 9.1, we can assume that $\mathscr{Z}$ is reflexive. Let $T$ be a continuous linear operator from $\mathscr{Z}$ onto $l^{p}$, such that $\|T\|=1$. The jth component of $T(x)$ will be denoted by $L_{j}(x)$, so that $T(x)=\sum L_{j}(x) e_{j}$. Define

$$
f(x)=\sum_{j=1}^{\infty} L_{j}(x)^{N},
$$

where $N$ is an integer satisfying $N \geq p$. One checks that $f$ is a pointwise limit of its partial sums, so that $f \in A_{p b}(B)$. On account of the open mapping principle, there exists $M>0$ so that each $e_{j}$ is of the form $T\left(z_{j}\right)$ for some $z_{j} \in \mathscr{Z}$ satisfying $\left\|z_{j}\right\| \leq M$. Set $y_{j}=z_{j} /(2 M)$. Then $f\left(y_{j}\right)=(1 / 2 M)^{N}$ is bounded away from 0 . On the other hand, if $z$ is any weak limit point of the sequence $\left\{y_{j}\right\}$, then $T(z)$ is a weak limit point of the sequence $e_{j} /(2 M)$, so that $T(z)=0$, and $f(z)=0$. Thus $f$ is not continuous with respect to the weak topology on $\frac{1}{2} B$, and by Lemma 8.2 $A(B)$ is not tight.

In closing, we mention the following criterion.

9.5. Theorem. If $A(B)$ is tight on $\bar{B}$, then each point of the unit sphere $\bar{B} \backslash B$ of $\mathscr{Z}$ is a (generalized) peak point for $A(B)$.

Proof. Let $z \in \bar{B} \backslash B$, and let $\mu$ be a representing measure on $\bar{B}$ for $z$. Then $\mu_{r}(E)=\mu(r E)$ defines a representing measure $\mu_{r}$ for $r z$, which converges weak-star to $\mu$ as $r \uparrow 1$. By [2], or Lemma 8.4, either $\mu=\delta_{z}$ or else $r z$ lies in the same Gleason part as $z$ eventually. The latter case is excluded, since $B$ forms by itself a Gleason part for $A(B)$. Hence the only representing measure for $z$ is the point mass $\delta_{z}$, and $z$ is a (generalized) peak point for $A$.

\section{REFERENCES}

1. E. L. Arenson, Gleason parts and the Choquet boundary of a function algebra on a convex compactum, Zap. Nauchn. Sem. Leningrad. Otdel. Mat. Inst. Steklov. (LOMI) 113 (1981), 204-207.

2. B. Cole and T.W. Gamelin, Tight uniform algebras, J. Funct. Anal. 46 (1982), 158-220.

3. - Weak-star continuous homomorphisms and a decomposition of orthogonal measures, Ann. Inst. Fourier (Grenoble) 35 (1985), 149-189.

4. D. W. Dean, The equation $L\left(E, X^{* *}\right)=L(E, X)^{* *}$ and the principle of local reflexivity, Proc. Amer. Math. Soc. 40 (1973), 146-148.

5. J. Diestel, Sequences and series in Banach spaces, Springer-Verlag, Berlin, 1984. 
6. _. A survey of results related to the Dunford-Pettis property, Proc. Conference on Integration, Topology and Geometry in Linear Spaces, W. Graves (ed.), Contemp. Math., Vol. 2, Amer. Math. Soc., Providence, R.I., 1980, pp. 15-60.

7. T. W. Gamelin, Uniform algebras, 2nd ed., Chelsea, New York, 1984.

8. __ Uniform algebras on plane sets, Approximation Theory, G. G. Lorentz (ed.), Academic Press, 1973, pp. 100-149.

9. J. Lindenstrauss and L. Tsafriri, Classical Banach spaces, Vol. 1, Springer-Verlag, Berlin, 1977.

10. J. E. Littlewood, On bounded bilinear forms in an infinite number of variables, Quart. J. Math. 1 (1930), 164-174.

11. L. Nachbin, Topology on spaces of holomorphic mappings, Springer-Verlag, Berlin, 1969.

12. A. Pelczynski, A property of multilinear operations, Studia Math. 16 (1957), 173-182.

13. G. Pisier, Factorization of linear operators and geometry of Banach spaces, CBMS Regional Conf. Ser. in Math., No. 60, Amer. Math. Soc., Providence, R.I., 1986.

14. H. R. Pitt, A note on bilinear forms, J. London Math. Soc. 11 (1936), 174-180.

Department of Pure Mathematics and Mathematical Statistics, Cambridge UniverSity, Cambridge, ENGland CB2 1SB

Department of Mathematics, Brown University, Providence, Rhode Island 02912

Department of Mathematics, University of California, Los Angeles, California 90024 